\title{
¿Que nos señala la literatura en relación al trasplante renal y embarazo?
}

\author{
Silvia Barrios Araya MSca, Miriam Ximena Concha MSc ${ }^{b}$. \\ ${ }^{a}$ Enfermera, ${ }^{b}$ Enfermera-Matrona. Escuela de Enfermería, Pontificia Universidad Católica de Chile.
}

\section{RESUMEN}

Antecedentes: La enfermedad renal crónica terminal es una patología asociada a condiciones de vida y hábitos de las personas que aumentará su incidencia a nivel mundial en toda la población incluso en mujeres en edad fértil. El trasplante renal considerado como terapia de sustitución renal, ha permitido a mujeres en edad fértil llevar a buen término la gestación. Chile, cuenta con reportes históricos publicados de embarazos en mujeres trasplantadas, Estados Unidos reporta al 2010 un total de 2.000 embarazos, con el $75 \%$ de recién nacidos vivos aproximadamente. Objetivo: Conocer la normalidad y desviaciones de salud en la mujer embarazada con trasplante renal y algunas recomendaciones que favorezcan el término satisfactorio de la gestación. Método: Búsqueda bibliográfica en MEDLINE, ProQuest, Scielo y el metabuscador Tripdatabase, seleccionándose para su análisis 32 artículos. Análisis del tema: No se reporta la necesidad de terapia dialítica durante la gestación en mujeres trasplantadas, ya que las cifras de creatinina permanecen estables con valores entre $1,14 \mathrm{mg} / \mathrm{dL}$ y $1,43 \mathrm{mg} / \mathrm{dL}$ hasta la primera mitad de la gestación. Se reporta complicaciones maternas como infecciones urinarias, preclampsia, hipertensión arterial crónica, y complicaciones neonatales como prematuridad, restricción del crecimiento intrauterino, aborto espontáneo y muerte neonatal, y complicaciones del injerto: rechazo al trasplante. Los estudios mencionan la utilización de esquemas de uno o más inmunosupresores, los cuales se mantuvieron o se modificaron de acuerdo a una medición basal al inicio del control prenatal. Conclusión: En general el embarazo en las mujeres trasplantadas tiene un buen resultado si reciben consejería y vigilancia multidisciplinaria.

\section{PALABRAS CLAVE: Embarazo, trasplante renal}

\section{SUMMARY}

Background: Chronic kidney disease is a terminal disease associated with living conditions and habits of the people will increase their incidence worldwide in the entire population including women of childbearing age. Kidney transplantation considered as renal replacement therapy, has allowed women of childbearing age to completing gestation. Chile, has published historical reports of pregnancies in transplanted women, United States reports for 2010 a total of 2,000 pregnancies, with $75 \%$ of newborns about living. Objective: To determine normal and pathologic processes in pregnant renal transplant and some recommendations that favor successful completion of pregnancy woman. Methods: Literature search was carried out in MEDLINE, ProQuest, Scielo and Tripdatabase metasearch, selecting 32 articles for analysis. Analysis of the theme: Not reported the need for dialysis therapy during pregnancy in women transplanted because creatinine levels remain stable with values between $1.14 \mathrm{mg} / \mathrm{dL}$ and $1.43 \mathrm{mg} / \mathrm{dL}$ until the first half of gestation. Reported maternal complications: urinary tract infections, preeclampsia, chronic hypertension; neonatal complications: prematurity, intrauterine growth restriction, spontaneous abortion and neonatal death and 
complications and graft: transplant rejection. Studies mentioned schemes the use of one or more immunosuppressive, which were maintained or modified according to a baseline measurement at the beginning of prenatal care. Conclusion: Overall pregnancy in women has transplanted a good result if you receive counseling and multidisciplinary monitoring.

\section{KEY WORDS: Pregnancy, kidney transplantation}

\section{INTRODUCCIÓN}

La enfermedad renal crónica terminal (ERCT) es una patología que de acuerdo a las condiciones de vida y hábitos de las personas se presume que seguirá incrementando su incidencia a nivel mundial en toda la población, incluso en mujeres en edad fértil con posibilidad de embarazo $(1,2)$.

La tasa de trasplante en Chile para el año 2013 fue de $13,8 \%$, año en el que se realizaron 243 trasplantes renales (3). El primer embarazo exitoso en una mujer trasplantada de riñón data del año 1958. Actualmente las mujeres receptores de un trasplante de riñón, toleran bien el embarazo llevando a término la gestación exitosamente, tanto para la madre, el neonato y el injerto (4). En relación a reportes de embarazo en mujeres trasplantadas, existen datos históricos en Chile con resultados materno-perinatal satisfactorios $(5,6,7,8)$.

El National Transplantation Pregnancy Registry de USA (NTPR) reportó al 2010 un total de 2.000 embarazos, con recién nacidos vivos cercano al $75 \%$, cifra similar a la población general (9).

En general, las mujeres en edad fértil con ERCT tienen una disfunción del eje hipotálamo-hipófisogonadal lo que se traduce en una infertilidad, la cual se revierte aproximadamente a los 6 meses postrasplante, momento en el cual se abren las posibilidades de concepción $(4,10)$. La tasa de embarazo después de un trasplante es entre el $5 \%$ y el $10 \%$, encontrando diversas complicaciones tanto maternas como neonatales y complicaciones propias del injerto $(4,11,12)$.

El objetivo de esta revisión bibliográfica es conocer la normalidad y desviaciones de salud en la mujer embarazada con trasplante renal y algunas recomendaciones que favorezcan el término satisfactorio de la gestación.

\section{METODOLOGÍA}

Se realizó una búsqueda en las bases de datos PUBMED, CINAHL (EBSCO), EBM Reviews (Ovid), ProQuest Nursing Journals \& Allied Health, entre agosto 2013 y marzo 2014. Se utilizó para la estrategia de búsqueda las palabras claves: pregnancy y kidney tranplantation y sus respectivas traducciones al momento de realizar la búsqueda en bases de datos en idioma español. Los criterios de selección de los artículos fueron investigaciones realizadas con mujeres embarazadas con trasplante renal, antigüedad de los artículos no mayor a 10 años a excepción de algunos que se consideraron relevantes para esta revisión. Se incluyeron artículos en idioma inglés, portugués y español. Se encontraron un total de 32 artículos que cumplían los criterios de inclusión para esta revisión. A continuación se presenta el análisis del tema que contempla los siguientes puntos: función renal antes, durante y pos embarazo; factores asociados al embarazo y parto en mujeres trasplantadas; complicaciones durante el embarazo; término y resolución del embarazo, y recomendaciones en relación a la fertilidad y embarazo.

\section{FUNCIÓN RENAL ANTES, DURANTE Y POST EMBARAZO}

Creatinina sérica. La creatinina sérica promedio previa al embarazo en mujeres trasplantadas, fue reportada por un número amplio de investigadores, con cifras promedios entre 1,1 y $1,3 \mathrm{mg} / \mathrm{dL}$ $(13,14,15,16)$. Los niveles de creatinina más baja fue la reportada por Keitel (2004) con un valor de $0,4 \mathrm{mg} / \mathrm{dL}$ y un máximo de $3,9 \mathrm{mg} / \mathrm{dL}$ en el estudio de Cruz y cols $(2007)(15,17)$. No se reporta la necesidad de terapia dialítica durante la gestación en mujeres trasplantadas, ya que las cifras de creatinina permanecen estables con valores promedio entre $1,14 \mathrm{mg} / \mathrm{dL}$ y $1,43 \mathrm{mg} / \mathrm{dL}$ hasta la primera mitad de la gestación $(13,14)$, período que coincide con la sobrecarga hídrica de una gestante normal (18). Sin embargo, esta condición de estabilidad se pierde previo al parto, encontrándose cifras por sobre los $2,0 \mathrm{mg} / \mathrm{dL}$ (18).

En un embarazo bien controlado no se verá afectada la función renal, siempre y cuando los niveles de creatinina sérica se mantengan bajo un valor de $1,5 \mathrm{mg} / \mathrm{dl}$ pregestacional (4). Por consiguiente el injerto renal estable no constituye necesariamente una contraindicación para el embarazo, no así, en aquellas mujeres que presenten creatininas séricas mayores a $2,5 \mathrm{mg} / \mathrm{dl}$ previo a la gestación, ya que tienen un alto riesgo de sufrir un rechazo renal durante el embarazo (12). Un estudio con una casuística importante mostró que la función del injerto renal post embarazo exitoso fue reportada en 133 mujeres con creatinina promedio de 
$1,84 \mathrm{mg} / \mathrm{dL}$ (rango: 0,51-2,9 mg/dL). En 31 mujeres $(23,3 \%)$ la función del injerto disminuyó después del trabajo de parto; 13 de ellas $(9,7 \%)$ perdieron el injerto; en 8 de estas $(61,5 \%)$ la pérdida del injerto ocurrió dentro de los 3 años posterior al término del embarazo, mientras que en $5(38,5 \%)$ ocurrió 10 años posterior al parto. En el primer grupo, la sobrevida del injerto puede haber sido afectada por el embarazo (20).

Proteinuria. En relación a la presencia de proteínas en la orina, Cruz y cols (15), reportan en su estudio que un número reducido de mujeres inicia el embarazo con proteinuria positiva, que puede variar en un rango de 0,4 a 3,0 g/24 h, cifras que se ven incrementadas al momento del parto, pudiendo alcanzar pérdidas de proteínas de hasta 5,6 g/24 h, situación que en este grupo de mujeres puede ser explicada por el síndrome hipertensivo del embarazo (SHE) $(14,17)$.

\section{FACTORES ASOCIADOS AL EMBARAZO EN MUJERES TRASPLANTADAS}

Intervalo entre trasplante y embarazo. La función ovárica de la mujer trasplantada se recupera a los pocos meses de realizado el trasplante, esto se traduce en la posibilidad real de un embarazo en mujeres en edad fértil. La mayoría de los centros sugiere que el embarazo debe retrasarse idealmente entre 18 a 24 meses pos trasplante $(21,22)$. A pesar de esta recomendación, los resultados de las investigaciones revisadas, reportan un rango mayor de intervalo de tiempo entre el trasplante y el embarazo reportando hasta cuatro años (1317,19,23-27).

Tipo de injerto. El origen del donante no tiene implicancias en la fertilidad ni en la evolución de la gestación. La literatura hace referencia que la primera fuente de injerto utilizado en las mujeres fue donante vivo, el cual podía ser relacionado o no $(13,15,19,23-25)$, este hallazgo difiere de Ventura y cols (28) y Queipo y cols (29), quienes reportan que los injertos de las mujeres trasplantadas provenían mayoritariamente de donante cadáver.

Enfermedades previas al embarazo. La mayoría de los estudios, reportan como desviaciones de salud a considerar en la planificación de cuidados profesionales previo a la gestación, la hipertensión arterial (HTA), la diabetes mellitus (DM) y la glomerulonefritis. Se sabe que en condiciones normales del embarazo existe una sobrecarga de volumen de aproximadamente un 50\% al compararla con su condición pregestacional (30), estado que debe ser considerado en mujeres trasplantadas, dado que existe un alto riesgo de compromiso del injerto en mujeres que se embarazan (4). Durante la gestación se deberá ser cauto en el control de la presión arterial (PA) y proteinuria, ya que ambas se detectan en un tercio de las mujeres trasplantadas, cifra muy superior a la población general. Cabe mencionar que el SHE aumenta el riesgo de la presencia de proteínas en la orina, restricción de crecimiento intrauterino y muerte materna. De acuerdo a la información proporcionada por el Centro de Diagnóstico e Investigaciones Perinatales (CEDIP), el SHE comparte con el aborto séptico el primer lugar entre las causas de mortalidad materna en Chile (31). Se identificó en esta revisión como una patología relevante en mujeres trasplantadas la HTA crónica, sea ésta diagnosticada o tratada previa al embarazo o bien pesquisada al inicio del control prenatal, ya que si la HTA se manifiesta después de la semana veinte de la gestación, es considerada SHE (14). En relación a lo anterior, varios autores reportan una incidencia cercana al $80 \%$ de HTA no controlada al momento de la concepción $(14,17,18,32)$, situación que difiere de Cruz y cols (15), quien reporta que un $45,3 \%$ de las mujeres de su estudio presentaban HTA pregestacional en tratamiento con antihipertensivos. Las mujeres trasplantadas que se embarazan y que inician su gestación con HTA no diagnosticada, pueden llevar a enmascarar un detrimento de la función del injerto ya sea por un daño propio o por el compromiso renal que causa el SHE (21). También existen otras condiciones patológicas referidas en la literatura que ocupan los primeros lugares entra las que se encuentran la DM pregestacional y la glomerulonefritis crónica, esta última con cifras cercanas al $60 \%$ $(11,14,16,18,23,25-27)$. Otras que se ven con menor incidencia, son la nefropatía de reflujo, enfermedad de riñón poliquístico dominante del adulto, nefropatía intersticial, síndrome nefrótico, nefritis de Henoch-Schonlein e hidronefrosis $(19,25,26)$.

Terapia inmunosupresora y embarazo post trasplante renal. En cuanto a la inmunosupresión, el uso de inmunosupresores se ha asociado a malformaciones estructurales en el feto, las que se presentan con una incidencia de aproximadamente un $4,5 \%$, similar a la población general (4).

Todos los estudios revisados reportan la utilización de esquemas de uno o más inmunosupresores, los cuales se mantuvieron o se modificaron de acuerdo a una medición basal al inicio del control prenatal y mediciones posteriores durante el embarazo $(13,15,16,20,23,33)$. La combinación de inmunosupresores más utilizada fue azatioprina, ciclosporina y prednisona/prednisolona $(14,16,17,25)$. La inmunosupresión debe mantenerse igual a excepción de aquellas usuarias que utilicen ciclosporina A (CsA), ya que ésta requiere aumentar la dosis para lograr niveles terapéuticos, en cuanto a la indicación del micofenolato mofetil se sugiere suspender antes de la concepción, ya que se le han atribuido algunas malformaciones asociadas a su uso (4).

Antecedentes de embarazo previo al trasplante. En relación al número de embarazo previo al tras- 
plante, esto fue reportado por Miranda y cols (33), quien en su estudio señala que el $59 \%$ de las mujeres tuvo un promedio de dos embarazos previos al trasplante, situación que es importante de considerar ya que el embarazo sensibiliza a las mujeres induciendo el desarrollo de anticuerpos a-HLA, que la constituyen en un paciente de alto riesgo inmunológico ya que pueden incurrir en el rechazo del injerto postrasplante a pesar de que durante el embarazo la capacidad de la respuesta inmune esta disminuida, por consiguiente, el rechazo del órgano debiese ser menor (4).

\section{COMPLICACIONES DURANTE EL EMBARAZO}

Los estudios analizados reportan en este grupo de mujeres diversas complicaciones que se presentan en orden de magnitud en complicaciones maternas: infecciones urinarias $35-46 \%$, preclampsia (PE) 30-50\%, hipertensión arterial crónica 5\%; complicaciones neonatales: prematuridad $50 \%$, restricción del crecimiento intrauterino 30-50\%, aborto espontáneo 30\% y muerte neonatal 5\%, y complicaciones del injerto como rechazo al trasplante entre $5-10 \%(4,11,12)$.

Infección del tracto urinario. Un estudio realizado en Chile en el año 2008, reportó como uno de los problemas de salud más frecuentes durante el embarazo, las infecciones urinarias, presentándose entre el 17 y $20 \%$ de las embarazadas sanas (34). Al comparar estas cifras con los resultados encontrados por otros autores en la literatura internacional, se puede ver que la incidencia abarca rangos más amplios, situándolos entre un 10 y $46 \%$ en mujeres embarazadas trasplantadas $(15,23,24,26,27)$.

Anemia. Es una complicación que varía con frecuencia entre los estudios revisados, Vásquez y cols (12) reportan un 33,3\% de los casos, a diferencia de Yassaee y cols (24) quienes estudiaron 95 casos de embarazos con un $65,3 \%$ de embarazadas con anemia.

Síndrome hipertensivo del embarazo. Se considera hipertensa independiente de la etiología, a la embarazada con cifras tensionales iguales o mayores de $140 / 90 \mathrm{mmHg}$ en dos tomas separadas por 6 horas de observación en reposo, cifras de $160 / 110 \mathrm{mmHg}$ o más, o HTA en rango menor a esta cifra asociada a proteinuria, hacen el diagnóstico de SHE y no requiere de mediciones repetidas, separadas en el tiempo (31). La HTA inducida por el embarazo se clasifica asimismo en PE y Eclampsia (E), la primera corresponde a la HTA inducida por el embarazo, que afecta de preferencia a primigestas, sin antecedentes cardiovasculares o renales, aparece en la segunda mitad de la gestación, se asocia a albuminuria y se caracteriza por ser reversible en el posparto. La E es la forma más severa de PE, donde la magnitud del alza tensional y del daño endotelial provoca una encefalopatía hiper- tensiva capaz de producir un cuadro convulsivo y/o coma en ausencia de patología neurológica previa, se presenta en aproximadamente el 0,1 a $0,3 \%$ de los partos (31). La HTA crónica es la elevación tensional detectada antes del embarazo o antes de las 20 semanas de gestación y que persiste en el postparto alejado. La hipertensión transitoria son alzas tensionales durante el peri parto y que revierten en el puerperio inmediato (31). Al igual que en poblaciones de mujeres embarazadas sanas, el SHE fue la segunda complicación más frecuente del embarazo $(15,31)$. La literatura revisada en relación al tema, reporta cifras más elevadas encontrando una estadística entre el 12 y $47,4 \%$ de las mujeres trasplantadas embarazadas, cifra superior a la reportada a la población de mujeres sanas, cuyo rango fluctúa entre un 10 y $20 \%(14,15,17,23-27,31,32)$. En el estudio de Cruz y cols (15), se reporta una incidencia de un 29,3\%. De estas embarazadas con SHE un $27,3 \%$ presentaron una preclampsia severa (PES) con compromiso renal (proteinuria y creatinina sérica elevada), que requirieron manejo en $\mathrm{UCl}$ en el periodo de pre y posparto.

Complicaciones neonatales. La literatura reporta datos aislados en relación a otras complicaciones del embarazo, tales como abortos espontáneos, parto de pretérmino, restricción del crecimiento intrauterino (RCIU), lo que dificulta realizar un análisis relacionado a estas complicaciones, sin embargo, se deben tener presentes, ya que son problemas que se presentan en gestantes de alto riesgo y que requieren de un manejo integral otorgado por profesionales expertos en el área (13-15,17,23,25-27).

Pérdida de injerto. Como ya se hizo mención, el embarazo en si en mujeres trasplantadas no necesariamente conlleva compromiso del injerto, especialmente si los niveles de creatinina se mantienen dentro de rangos normales para el embarazo (12). Sin embargo, de sobrepasar estos límites se podría acelerar el proceso de deterioro de la función renal lo que determina por un lado reiniciar la terapia dialítica después del puerperio o perder progresivamente el injerto en un lapso de 2 años posterior al embarazo $(13,15,19,23,24,27)$. La literatura recomienda el uso de antibióticos profilácticos y corticoides vía endovenosa cada 6 u 8 horas durante el trabajo de parto y en las primeras 24 a 48 horas del puerperio, con la finalidad de evitar el rechazo renal agudo posparto (4).

\section{TÉRMINOY RESOLUCIÓN DEL EMBARAZO}

En general el embarazo en las mujeres trasplantadas tiene un buen resultado logrando llegar al término con feto vivo en cifras cercanas al $80 \%$ de los casos reportados en la literatura (13-15,17,23$27,32)$. En un porcentaje no despreciable también se reporta que la tasa de aborto espontáneo varia en un rango entre un 8 y $28 \%(14,15,17,23,24)$. A 
pesar de que literatura recomienda el parto vaginal y sugiere remitir la cesárea solo a razones obstétricas justificadas (4), la revisión mostró que la vía de resolución del embarazo fue predominantemente la cesárea, ya sea electiva o de urgencia, asociada a una patología obstétrica, deterioro de la función renal materna o una disfunción placentaria aguda $(13,15,19,23,24,26,27)$.

\section{RECOMENDACIONES EN RELACIÓN A LA FER- TILIDAD Y EMBARAZO}

De acuerdo a la revisión de la bibliografía es importante recalcar y hacer visible el estrés que sufre el injerto durante la gestación, por ende, el equipo multidisciplinario con el cual se relaciona la mujer trasplantada en edad fértil debiese contemplar la consejería o asesoramiento preconcepcional durante el período pre y pos trasplante, la cual debe ser cautelosa en las propuestas relacionadas con la concepción y anticoncepción $(4,35,36)$. Si la mujer desea indeclinablemente embarazarse, la recomendación es que lo haga durante su terapia dialítica en pro de lograr el máximo de vida útil del riñón trasplantado, sin desconocer los riesgos que esto implica. Si no es así, según las recomendaciones dadas por el Report on the AST Consensus Conference on Reproductive Issues and Transplantation como las guías Europeas, la mujer portadora de un injerto renal que se embarace debe cumplir con los siguientes criterios: función renal estable con creatinina menor a $1,5 \mathrm{mg} / \mathrm{dl}$, sin episodio de rechazo agudo en los últimos 6 meses, presión arterial normal o bajo control solo con un fármaco antihipertensivo, proteinuria menor a 0,5 g/día, ecografía del injerto renal normal y tratamiento inmunosupresor recomendado $(37,38)$.

\section{CONCLUSIONES}

El trasplante renal le ha dado a la mujer con ERCT la posibilidad de mejorar su calidad de vida y asimismo la oportunidad de mejorar su vida sexual y reproductiva. Por consiguiente, de acuerdo a la revisión realizada es importante que la enfermera o enferma matrona conozca la fisiopatología renal y obstétrica y las posibles complicaciones de una mujer trasplantada que se embaraza. Con relación a la función renal peri-embarazo parece fundamental que este profesional conozca los rangos de creatinina y proteinuria ya que juegan un rol importante en el pronóstico de la función renal durante la gestación y en la sobrevida del injerto. Los valores recomendados de creatinina y proteinuria son cifras bajo 1,5 mg/dl y entre 0,4 a 3,0 g/24 horas, respectivamente. Así mismo, se pudo identificar dentro de los factores de riesgo asociados al embarazo y parto en mujeres trasplantadas la ocurrencia del embarazo antes de los dos años de ocurrido el trasplante, de igual forma la desviación de salud previa al embarazo más importante a considerar en la mujer gestante trasplantada es la hipertensión arterial en estado de cronicidad. Los antecedentes de embarazo previo al trasplante se tornan un tema significativo ya que el embarazo por sí mismo sensibiliza a las mujeres transformándolas en pacientes de alto riesgo inmunológico. En cuanto a la terapia inmunosupresora durante el embarazo habitualmente requiere de la asociación de al menos dos inmunosupresores, cuyo control y seguimiento deben ser rigurosos. De las complicaciones la PE resulta ser la más importante a partir de la segunda mitad del embarazo, ya que puede evolucionar a un cuadro grave con compromiso del sistema nervioso central y determinar la interrupción prematura del embarazo. En vista de lo revisado, sería apropiado promover la consejería preconcepcional relacionada con fertilidad y embarazo para mujeres con ERCT que se encuentre en tratamiento dialítico y que desea embarazarse y para la madre trasplantada en control prenatal, con énfasis en un enfoque de riesgo, de tal forma de acogerse a las recomendaciones internacionales al respecto. En caso de embarazadas trasplantadas, los controles de salud de éstas deben realizarse en un centro de nivel secundario en coordinación con el nivel primario de salud, que contemple un equipo multidisciplinario con obstetra, neonatólogo, trasplantólogo, nefrólogo, enfermera, matrona, psicólogo y nutricionista. Este equipo debe planificar, coordinar y ejecutar acciones que aseguren: los requerimientos del binomio madre-hijo, una adecuada evolución del embarazo, el crecimiento y desarrollo apropiado del feto y una óptima función del injerto renal, con la finalidad de pesquisar precozmente cualquiera desviación de salud y la toma decisiones en forma oportuna de manera tal, de asegurar el mínimo riesgo al cual puedan estar expuesta la madre, su feto y el injerto.

Finalmente es importante destacar que el rol de la enfermera nefrológica es realizar una valoración integral con enfoque de riesgo poniendo énfasis en monitorear los niveles de creatinina de la embarazada trasplantada, los niveles de presión arterial y niveles de anticuerpo. Asimismo, ésta deberá mantener una comunicación y coordinación expedita con la matrona o los equipos de obstetricia y neonatología con el fin de estar informados de la evolución y posibles complicaciones del binomio madre-hijo.

\section{REFERENCIAS}

1. Poblete H. XXXIII Cuenta de hemodiálisis crónica en Chile 2013 [Internet]. Sociedad Chilena de Nefrología. Disponible en: www.nefro.cl. Acceso 2 de Marzo de 2014.

2. Ortiz M, Valenzuela M. Informe de diálisis perito- 
neal 2013[Internet]. Sociedad Chilena de Nefrología. Disponible en: http://webcache.googleusercontent.com/search?q=cache:9rBblaYJaQ8J:www. asodi.cl/inf\%25C3\%25B3rmate-y-comparte/ biblioteca/cuenta-hemodialisis/finish/6-cuentas-hemodialisis/64-informe-dialisis-peritoneal$2013+\& c d=1 \& h l=e s \& c t=c l n k \& g l=c l$. Acceso 2 de Marzo de 2014.

3. Instituto de Salud Pública. Registro nacional de trasplante de órganos sección de histocompatibilidad, Sub. Depto de Inmunología ISP, 2013. Disponible en: http://www.ispch.cl/sites/default/files/documento/2010/04/Registro\%20Nacional\%20de\%20Trasplante $\% 20$ de $\% 20 \%$ C3\%B3rganos\%20Informe $\% 20$ a\%C3\%B10\%202012.pdf. Acceso 15 de Marzo de 2014.

4. Pefaur J, Rosati MP, Zehnder C, Toro J, Ortiz AM, Elgueta S, Kompatzky A, Espinoza O, Ogrodnik MT. Trasplante Renal. Guías Clínicas Sociedad Chilena de Trasplante 2010;6:85-8.

5. Donoso E, Orphanopoulos D, Fernández C, Barrena N, Vacarezza A, Valdés G, Martínez P, Martínez L. Homotrasplante renal y embarazo. Rev Chil Obstet Ginecol 1883;48(4):228-37.

6. Molina R, Hasbún J, Van Cauwelaert R. Trasplante renal y embarazo. Tres casos clínicos de donante vivo. Rev Chil Obstet Ginecol 1983;8(4):238-43

7. Oyarzún E, Guardiola M, Mondión M, Valdés G, Pinto C, Vacarezza A. Rev Méd Chile 1993;121(12):13827. Trasplante renal y embarazo: experiencia en 6 pacientes.

8. Perucca E, Fernández A, Barra A, Franchi P, Torres J, James E. Aspectos obstétricos y perinatales en trasplante renal. Rev Chil Obstet Ginecol 1994;59;(4):27579.

9. Coscia LA, Constantinescu S, Moritz MJ, Frank AM, Ramirez CB, Maley WR, et al. Report from the National Transplantation Pregnancy Registry (NTPR): outcomes of pregnancy after transplantation. Clin Transpl 2010:65-85

10. Pallardó LM, Crespo JF. Embarazo y trasplante renal: luces y sombras. Nefrología 2008;28:148-50.

11. Romero JF, Ayala JA, Jiménez G. Embarazo en pacientes con trasplante renal: morbilidad materna y fetal. Ginecol Obstet Mex 2008;76:643-51.

12. Vásquez J, Ríos A. Complicaciones perinatales en mujeres con trasplante renal. Nefrología 2012;32:639-46.

13. Basaran O, Emiroglu R, Secme S, Moray G, Haberal M. Pregnancy and renal transplantation. Transplant Proc 2004;36:122-4.

14. Yildirim Y, Uslu, A. Pregnancy in patients with previous successful renal transplantation. Int J Gynaecol Obstet 2005;90:198-202.

15. Cruz Lemini MC, Ibarguengoitia Ochoa F, Villanueva Gonzalez MA. Perinatal outcome following renal transplantation. Int J Gynaecol Obstet 2007;96:76-9.

16. Fischer T, Neumayer HH, Fischer R, Barenbrock, M, Schobel H, Lattrell B, et al. Effect of pregnancy on long-term kidney function in renal transplant recipients treated with cyclosporine and with azathioprine. Am J Transplant 2005;5:2732-9.

17. Keitel E, Bruno RM, Duarte M, Santos AF, Bittar AE, Bianco PD, et al. Pregnancy outcome after renal transplantation. Transplant Proc 2004;36:87-1.

18. Lapidus AM. Cambios fisiológicos maternos durante el embarazo. En: Perez A, Donoso E. (Eds). OBSTETRICIA. 3a ed. Santiago: Mediterráneo; 1999. pp 182183.

19. Kurata A, Matsuda $\mathrm{Y}$, Tanabe K, Toma H, Ohta $\mathrm{H}$. Risk factors of preterm delivery at less than 35 weeks in patients with renal transplant. Eur J Obstet Gynecol Reprod Biol 2006;128:64-8.

20. Toma H, Tanabe K, Tokumoto T, Kobayashi C, Yagisawa $T$. Pregnancy in women receiving renal dialysis or transplantation in Japan: A nationwide survey. Nephrol Dial Transplant 1999;14:1511-16.

21. Bar J, Ben-Rafael Z, Padoa A, Orvieto R, Boner G, Hod $M$. Prediction of pregnancy outcome in subgroups of women with renal disease. Clin Nephrol 2000;53:43744.

22. Toirac A, Pascual V. El riñón y el aparato excretor urinario en la embarazada. Consideraciones básicas. MEDISAN 2013;17:357-83.

23. Kashanizadeh N, Nemati E, Sharifi-Bonab M, Moghani-Lankarani M, Ghazizadeh S, Einollahi B, et al. Impact of pregnancy on the outcome of kidney transplantation. Transplant Proc 2007;39:1136-8.

24. Yassaee F, Moshiri F. Pregnancy outcome in kidney transplant patients. Urol J 2007;4:14-7.

25. Rahamimov R, Ben-Haroush A, Wittenberg C, Mor E, Lustig S, Gafter U, et al. Pregnancy in renal transplant recipients: Long-term effect on patient and graft survival. A single-center experience. Transplantation 2006;81:660-4.

26. Pezeshki M, Taherian AA, Gharavy M, Ledger WL. Menstrual characteristics and pregnancy in women after renal transplantation. Int $\mathrm{J}$ Gynaecol Obstet 2004;85:119-25.

27. Ghanem ME, El-Baghdadi LA, Badawy AM, Bakr MA, Sobhe MA, Ghoneim MA. Pregnancy outcome after renal allograft transplantation: 15 years' experience. Eur J Obstet Gynecol Reprod Biol 2005;121:178-81.

28. Ventura A, Martins L, Dias L, Henriques AC, Sarmento AM, Braga J, Pereira MC, Guimaraes S. Pregnancy in renal transplant recipients. Transplant Proc 2000;32:2611-2

29. Queipo-Zaragoza JA, Vera-Donoso CD, Soldevila A, Sanchez-Plumed J, Broseta-Rico E, Jimenez-Cruz JF. Impact of pregnancy on kidney transplant. Transplant Proc 2003;35:866-7.

30. Gómez R, Nazar C. Consideraciones generales de la embarazada con enfermedad cardiaca congénita y adquirida. Rev Chil Anest 2013;42:77-87

31. Centro de Diagnóstico e Investigaciones Perinatales (CEDIP). Guía Perinatal: Síndrome hipertensivo del embarazo. Disponible en: http://www.cedip.cl/Guias/ Guia2003/capitulo13.swf. Acceso 22 de enero de 2014. 
32. Naqvi R, Noor $\mathrm{H}$, Ambareen $\mathrm{S}$, Khan $\mathrm{H}$, Haider $\mathrm{A}$, Jafri $\mathrm{N}$, et al. Outcome of pregnancy in renal allograft recipients: SIUT experience. Transplant Proc 2006;38:2001-2.

33. Miranda C, Melaragno C, Camara NO, Pacheco-Silva $A$, Medina-Pestana PJ. Adverse effects of pregnancy on renal allograft function. Transplant Proc 2002;34:506-7.

34. Sandoval J, Fica A, Caballero R. Tratamiento y profilaxis antibiótica de patologías comunes en ginecología obstetricia. Rev Hosp Clín Univ Chile 2008;19:245-69.

35. Díaz JM, Canal C, Giménez I, Guirado L, Facundo C, Solá R, Ballarín J. Embarazo en receptoras de trasplante renal: efectos en la madre y el niño. Nefrología 2008;28:174-7.
36. Fuchs K, Wu D, Ebcioglu Z. Pregnancy in renal transplant recipients. Sem Perinat 2007;31:339-47.

37. McKay DB, Josephson MA, Armenti VT, August P, Coscia LA, Davis CL, et al. Reproduction and transplantation: report on the AST Consensus Conference on Reproductive Issues and Transplantation. Am J Transplant 2005;5:1592-9.

38. EBPG Expert group in renal transplantation. European best practice guideline for renal transplantation. Section IV. Long-term management of the transplant recipient. IV. 10. Pregnancy in renal transplant recipient. Nephrol Dial Tranplant 2002;17(suppl 4):50-5. 